\title{
O ENSINO DE REAÇÕES ORGÂNICAS USANDO QUÍMICA COMPUTACIONAL: I. REAÇÕES DE ADIÇÃO ELETROFÍLICA A ALQUENOS
}

\author{
Arquimedes Mariano, Elizete Ventura, Silmar A. do Monte, Claudia F. Braga, Antônio B. Carvalho e Regiane C. M. \\ U. Araújo* \\ Departamento de Química, Universidade Federal da Paraíba, 58036-300 João Pessoa - PB, Brasil \\ Otávio L. Santana \\ Unidade Acadêmica de Educação, Universidade Federal de Campina Grande, Campus Cuité - PB, Brasil
}

Recebido em 30/10/06; aceito em 26/10/07; publicado na web em 9/4/08

\begin{abstract}
TEACHING ORGANIC REACTIONS USING COMPUTATIONAL CHEMISTRY: I. ELECTROPHILIC ADDITION REACTIONS TO ALKENES. Basic concepts that play an important role in some organic reactions are revisited in this paper, which reports a pedagogical experience involving undergraduate and graduate students. A systematic procedure has been applied in order to use widespread available computational tools. This paper aims to discuss the use of computers in teaching electrophilic addition reactions to alkenes. Two classical examples have been investigated: addition to non-conjugated alkenes and addition to conjugated dienes. The results were compared with those normally discussed in organic textbooks. Several important concepts, such as conformational analysis and energy control (kinetic and thermodynamic) involved in reaction mechanisms can be taught more efficiently if one connects theoretical and practical tools.
\end{abstract}

Keywords: organic mechanism teaching; electrophilic reaction; quantum chemistry.

\section{INTRODUÇÃO}

O ensino dos mecanismos de reações em disciplinas de Química Orgânica para alunos dos cursos de graduação e pós-graduação em Química ainda representa um desafio. De certa forma, as idéias dos alunos sobre reações orgânicas estão relacionadas à "intuição química" e refletem um conhecimento compartimentalizado. O entendimento de uma reação química envolve conceitos microscópicos (em nível molecular) e macroscópicos (coleção de muitas moléculas) dos sistemas químicos investigados (reagentes, estados de transição e produtos) e o que se espera é que o aluno consiga correlacionar estes conceitos. Os principais fatores macroscópicos que influenciam o curso das reações são essencialmente dois: a variação da energia livre do sistema (que atua no controle termodinâmico da reação) e a velocidade de conversão de reagentes em produtos (relacionada ao controle cinético). Considerando a estrutura molecular, é possível fazer uma abordagem enfocando conceitos fundamentais, como comprimentos de ligações, ângulo de torção, análise conformacional, superfícies de energia potencial, estruturas de mínimo e máximo de energia, estrutura do estado de transição e modos vibracionais relacionados à quebra e formação de ligações. A aprendizagem destes conceitos inter-relacionados conduz a uma compreensão da química na sua integralidade, e não apenas a uma compreensão fragmentada do conhecimento.

Uma vez que o ensino é um processo dinâmico que exige envolvimento e constante reflexão sobre as várias etapas do processo, algumas vezes se fazem necessárias mudanças de paradigmas para que se possam conduzir práticas docentes que promovam uma aprendizagem mais eficiente pelos alunos. Este processo de construção pode ser feito por uma via dupla, aliando teorias a ferramentas computacionais. Dessa forma, há uma fundamentação capaz de permitir a compreensão de um fenômeno (teoria) e um instrumento (computador) facilitando esta compreensão, sem despre-

*e-mail: regiane@quimica.ufpb.br zar a relevância do professor como mediador do processo.

Uma das formas de facilitar o complexo processo da aprendizagem de conceitos, por parte dos alunos, é torná-lo sistemático, embora esta não seja uma tarefa fácil. Desta forma, os recentes avanços nos programas de Química Computacional têm possibilitado mudanças significativas no Ensino de Química. ${ }^{1}$ A Química Quântica Computacional facilita a compreensão de conceitos fundamentais, proporcionando condições aos alunos para que o aprendizado se torne mais efetivo, não desprezando a "intuição química", mas fundamentado-a com argumentos mais concretos.

O objetivo do presente trabalho é mostrar como a química quântica computacional, juntamente com programas adequados de modelagem e visualização molecular, pode ser uma grande aliada no entendimento de mecanismos de reações orgânicas. Foram escolhidas reações clássicas de química orgânica, descritas nos livros-texto. ${ }^{2}$

O estudo foi simplificado assumindo que o mecanismo ocorre de forma concertada (em uma única etapa). Esta aproximação simplifica o procedimento computacional, uma vez que evita a necessidade de inclusão do meio reacional (efeitos do solvente) nos cálculos, pois o mecanismo não-concertado envolve a formação de um carbocátion, o qual necessita de um solvente polar para a sua estabilização. O desafio se constitui em mostrar que é possível utilizar um método ab initio de estrutura eletrônica simples, como, por exemplo, o método Hartree-Fock com uma base mínima (STO$3 \mathrm{G})$, na construção de um modelo que sirva como ferramenta para auxiliar no entendimento de alguns conceitos fundamentais relacionados aos mecanismos de reações. Por se tratar de um modelo matemático, ou seja, de uma representação simplificada da realidade, tudo o que se espera é descrever corretamente, ao menos qualitativamente, algum aspecto de interesse particular do problema sob estudo, de modo semelhante ao que é feito em outros trabalhos na área de educação. ${ }^{3}$ A escolha deste nível de cálculo foi baseada na necessidade de minimizar o tempo computacional necessário para executar todas as etapas envolvidas no estudo da rea- 
ção. Dessa forma, é possível discutir todos os aspectos do tratamento computacional, desde a construção das entradas para os cálculos até a análise final dos resultados, em uma única aula expositiva de aproximadamente $2 \mathrm{~h}$, usando um sistema multimídia composto por datashow e computador (que, para tal finalidade, não precisa possuir muitos recursos; neste trabalho o equipamento utilizado possui um processador AMD de $1 \mathrm{GHz}$, com $256 \mathrm{Mb}$ de RAM e HD de $40 \mathrm{~Gb}$ ).

É importante salientar que o presente artigo se baseia em uma experiência didática real, onde, além dos principais aspectos do mecanismo da reação orgânica, conforme descritos nos livros-texto, foi feita uma breve revisão sobre a aplicabilidade dos principais métodos de estrutura eletrônica. Foram salientadas as necessidades da inclusão de correlação eletrônica (necessária para a descrição da quebra e formação de ligações) e do uso de bases extensas (para a descrição de sistemas carregados) no estudo dos sistemas apresentados. Por outro lado, uma vez que o objetivo do trabalho é realizar todas as etapas do cálculo em uma única aula expositiva, a utilização de métodos correlacionados e bases extensas são inviáveis.

Sabe-se que a inclusão de correlação eletrônica pode mudar as posições relativas dos pontos estacionários de reagentes, produtos e estados de transição. Existem duas contribuições importantes para a correlação eletrônica. A primeira, denominada "correlação dinâmica", é importante nos casos em que o determinante de referência Hartree-Fock domina a expansão CI. A segunda, denominada "correlação estática", é relacionada à quase-degenerescência dos orbitais de fronteira, resultando em contribuições significativas de alguns determinantes excitados. Além disso, é importante salientar a natureza multiconfiguracional intrínseca da função de onda para a descrição de uma coordenada de reação. Uma descrição mais precisa das regiões da superfície de energia potencial relevantes ao estudo da quebra e formação de ligações químicas requer o emprego de metodologias adequadas de estrutura eletrônica que levem em conta o caráter multiconfiguracional do problema. Entre estes métodos pode-se destacar o MCSCF (Multiconfigurational SCF), nas suas diversas versões (CASSCF, RAS, etc.), que fornece resultados qualitativamente corretos para os casos onde a correlação estática é importante. A inclusão de correlação eletrônica dinâmica na função de onda é feita mediante a introdução de excitações até um determinado nível, a partir de um dado espaço de referência. O método CI multi-referência com excitações simples e duplas (MR-CISD), bem como suas variantes que incluem correções de extensividade (MR-CISD+Q e MR-AQCC), representam o estado da arte em termos de cálculos correlacionados. Também se pode mencionar o método CASSCF (Complete Active Space SCF) corrigido com teoria de perturbação de segunda ordem (CASPT2), que apresenta uma demanda computacional bem menor que o método MR-CISD.

Geometrias de estados excitados podem ser bem diferentes das do estado fundamental, como no caso da molécula do acetileno, ${ }^{4} \mathrm{O}$ que torna imprescindível a consideração do caráter multi-configuracional na função de onda. Entretanto, mesmo para o estado fundamental, nos casos onde há uma mudança muito grande nas configurações eletrônicas, o que normalmente ocorre quando se estuda um caminho de reação, a descrição correta dos pontos estacionários nas superfícies de energia potencial requer uma função de onda multiconfiguracional. É o que ocorre, por exemplo, no mecanismo de rearranjo de Cope para o 1,5 -hexadieno, ${ }^{5}$ nas conformações do tetrametileno ${ }^{6}$ e no mecanismo de Diels-Alder para adição de butadieno e etileno. ${ }^{7}$ Sendo assim, pode-se concluir que o caráter multiconfiguracional é a regra e não a exceção.

Entretanto, alguns aspectos importantes com respeito ao uso de métodos multiconfiguracionais e multireferência merecem des- taque, tais como as entradas normalmente não são simples; é necessário ter um controle muito grande das mudanças nas configurações eletrônicas, nos estados eletrônicos que estão sendo calculados e nas propriedades que se pretende obter; a demanda computacional é alta. Estes aspectos inviabilizam o uso desses métodos para diversas aplicações e os tornam impraticáveis para os objetivos da presente proposta.

\section{METODOLOGIA}

A metodologia adotada pode ser dividida nas seguintes etapas: breve revisão de química quântica, a partir de aulas expositivas, onde foram abordados os principais métodos de estrutura eletrônica, suas vantagens e desvantagens; estudo de alguns mecanismos clássicos de reações orgânicas, tais como reação de substituição nucleofílica, de eliminação e adição eletrofílica, discutindo-se os principais mecanismos envolvidos nestas reações e as condições que favorecem cada classe de reação; realização de cálculos de estrutura eletrônica objetivando o estudo dos mecanismos discutidos na etapa anterior, incluindo-se mecanismos alternativos para consolidar a fundamentação dos conceitos anteriores; interpretação dos resultados e comparação com a abordagem discutida nos livros-texto de química orgânica, possibilitando a revisão de conceitos fundamentais do mecanismo, baseando-se nos aspectos estereoquímicos e energéticos, a partir de uma análise termo-dinâmica e cinética da reação.

\section{PROCEDIMENTO COMPUTACIONAL}

Os programas utilizados para realização dos cálculos e preparação das entradas foram o Gaussian $98 \mathrm{~W}^{8}$ e o GausView 3.0. As etapas envolvidas no estudo dos mecanismos de reação foram: otimização completa das geometrias de reagentes e produtos; busca pelo estado de transição, usando a metodologia QST2 ou QST3, de acordo com a necessidade, ambas implementadas no programa Gaussian 98W; ${ }^{9}$ otimização completa da geometria do estado de transição e caracterização deste ponto estacionário, através da análise dos modos normais de vibração; cálculo da coordenada de rea-ção usando a implementação IRC (Intrinsic Reaction Coordinate $)^{10}$ do programa Gaussian.

Em todas as etapas anteriores foi utilizado o método HartreeFock $^{11}$ com a base STO-3G. ${ }^{12}$ Para a busca pela estrutura do estado de transição usando a opção QST2, as geometrias dos reagentes e produtos são incluídas em uma mesma entrada. Esta entrada pode ser preparada no GausView da seguinte forma: abre-se inicialmente a geometria otimizada do reagente e, em seguida, a do produto, observado-se a opção Target no menu Open Files, onde se deve escolher a opção Add all files to active molecule group. Dessa forma, têm-se as duas geometrias no mesmo arquivo. É importante que as duas geometrias tenham a mesma ordem de numeração dos átomos e conectividade nas duas geometrias de entrada. O próximo passo é escolher a opção QST2 para otimização, através da palavra-chave OPT=QST2. No GausView essa configuração pode ser obtida a partir do menu Calculate/Gaussian..., selecionando-se na página Job Type a opção Optimization e na opção Optimize to a selecionando-se o item "TS (QST2)". Quando a busca pelo estado de transição usando o método QST2 não funciona, a própria saída do cálculo sugere a busca com o QST3. Na opção QST3 é necessário incluir, além das estruturas do regente e do produto, uma terceira estrutura como "chute" inicial para o possível estado de transição. A entrada para este cálculo é feita de forma semelhante à opção QST2, apenas com a etapa adicional para a terceira estrutura e a palavra-chave OPT=QST3. 
A geometria do estado de transição, obtida com a metodologia QST2 ou QST3, deve ser otimizada incluindo-se o cálculo das constantes de força no início do procedimento, o que pode ser feito com a palavra-chave OPT=(TS,CALCFC). A caracterização do estado de transição é feita mediante um cálculo adicional de constantes de força, utilizando-se a palavra-chave FREQ. No GausView essa configuração pode ser obtida a partir do menu Calculate/ Gaussian..., selecionando-se na página Job Type a opção Opt+Freq, na opção Optmize to a selecionando-se o item TS (Berny) e na opção Calculate Force Constants selecionando-se o item Once. No resultado deste cálculo deve haver apenas uma frequiência imaginária. Na realidade, a frequiência é exibida com sinal negativo pelo programa, e é obtida em função da raiz quadrada da constante de força do modo normal característico do estado de transição. Esta constante de força, uma grandeza negativa, muda a natureza das forças harmônicas atuantes, que passam a ser denominadas auxiliadoras ao invés de restauradores, como na lei de Hook. No GausView o modo normal de freqüência imaginária pode ser visualizado a partir do menu Results/Vibrations... selecionando-se o primeiro modo normal indicado no diálogo Display Vibrations.

O único modo normal de frequiência imaginária, determinado na etapa anterior, caracteriza a estrutura como a de um estado de transição "legítimo". Este modo normal deve refletir o fato de que a estrutura obtida corresponde ao estado de transição da reação sob investigação, o que pode ser feito animando-se o modo normal na opção Start do diálogo Display Vibrations. No entanto, ainda é preciso confirmar esta análise preliminar, de modo a verificar se o estado de transição obtido é o "correto", efetuando-se um cálculo adicional para a determinação da coordenada de reação. A definição natural desta coordenada corresponde ao caminho descendente de energia mínima a partir do estado de transição, movendo-se em direção aos mínimos (reagentes e produtos) na superfície de energia potencial. Quando as coordenadas cartesianas ponderadas pelas massas são usadas, este caminho é também chamado "coordenada de reação intrínseca" (IRC). Este cálculo pode ser feito com a palavra-chave $\mathrm{IRC}=(\mathrm{CALCFC})$, podendo ser configurado com o GausView no menu Calculate/Gaussian..., selecionando-se na página Job Type a opção IRC, na opção Follow IRC selecionando-se o item Both directions e na opção Calculate Force Constants selecionando-se o item Once. O resultado deste cálculo pode ser visualizado a partir do menu Results/IRC....

\section{ADIÇÃO ELETROFÍLICA: FUNDAMENTAÇÃO TEÓRICA}

Uma generalização que permite prever a regiosseletividade das reações de adição de moléculas do tipo $\mathrm{X}-\mathrm{Y}$ a alquenos assimétricos de fórmula geral $\mathrm{R}_{2} \mathrm{CP}=\mathrm{CHR}$ é conhecida como regra de Markovnikov. Esta regra estabelece que a espécie parcialmente positiva do reagente $\mathrm{X}-\mathrm{Y}$ se liga ao átomo de carbono mais hidrogenado da ligação dupla (Esquema 1).

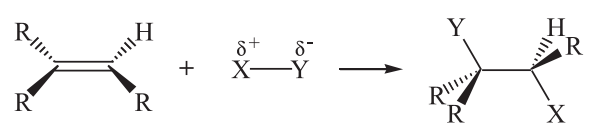

Esquema 1

Se a adição de um haleto de hidrogênio a um alqueno assimétrico resulta predominantemente em um de dois produtos possíveis, a reação é considerada regiosseletiva. A regiosseletividade pode ser parcial (2a) ou completa (2b), Esquema 2.

A regiosseletividade parcial é baseada na habilidade relativa dos átomos de carbono da ligação insaturada em acomodar a carga

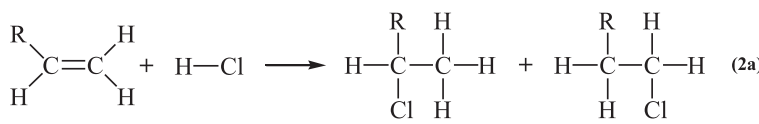

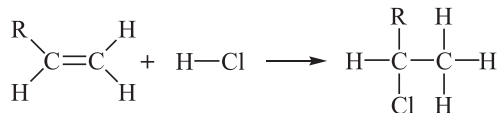

\section{Esquema 2}

positiva do carbocátion intermediário, formado após a adição do hidrogênio (Esquema 3).

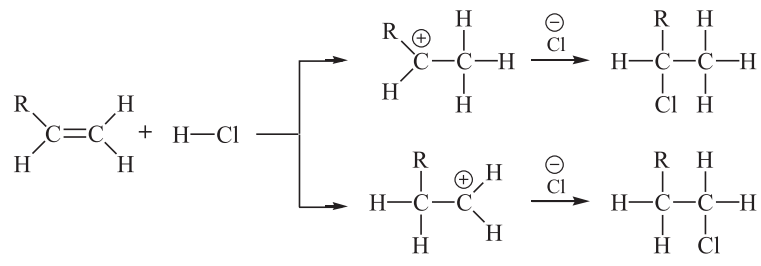

Esquema 3

É importante destacar que o entendimento do mecanismo de reações orgânicas não pode ser resumido em uma regra. Portanto, é inútil para o estudante decorar qualquer regra na ilusão de poder explicar a formação de um produto. Por exemplo, quando um alqueno assimétrico apresenta um grupo retirador de elétrons ligado a um dos carbonos da ligação insaturada, por exemplo, um íon amônio, a orientação da adição do haleto de hidrogênio ao alqueno é invertida. Dessa forma, o produto formado não segue a regra de Markovnikov (Esquema 4a):

$$
\left(\mathrm{CH}_{3}\right)_{3} \stackrel{\oplus}{\mathrm{N}}-\mathrm{CH}=\mathrm{CH}_{2} \stackrel{\mathrm{H}-\mathrm{Br}}{\longrightarrow}\left(\mathrm{CH}_{3}\right)_{3} \stackrel{\oplus}{\mathrm{N}}-\mathrm{CH}_{2}-\mathrm{CH}_{2}-\mathrm{Br}
$$

\section{Esquema $4 a$}

Esta inversão pode ser explicada com base na estabilidade do intermediário formado (Esquema 4b):

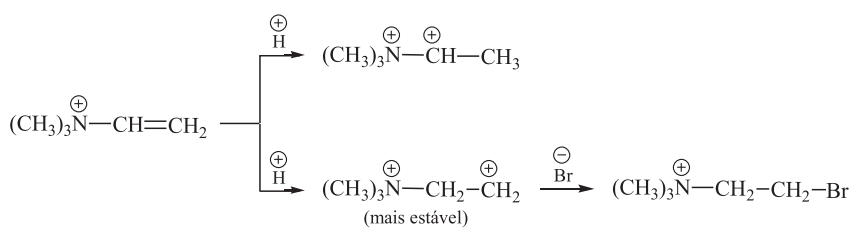

\section{Esquema $4 b$}

Portanto, a formação de um produto está intimamente relacionada à energética das espécies envolvidas, o que permite compreender os aspectos cinético e termodinâmico do mecanismo. Contudo, pode-se fazer uma releitura da regra de Markovnikov, fundamentando-a nessa nova abordagem.

\section{Adição a alquenos não-conjugados}

Produtos com estereoquímica predominantemente anti são formados a partir de reações de adição de reagentes do tipo $\mathrm{X}-\mathrm{Y}$ a alquenos não-conjugados. A estereoquímica destas reações pode ser influenciada pela temperatura e pelo solvente (Esquema 5).

A formação de produtos de adição com estereoquímica predominante anti pode ser explicada através de um mecanismo concertado, compatível com um processo termolecular cuja lei de velocidade é $v=k$ [Alqueno][HX] $]^{2}$ (onde $k$ corresponde à constante de velocidade e $v$ a velocidade da reação) (Esquema 6), ou através de um mecanismo iônico (Esquema 7). 


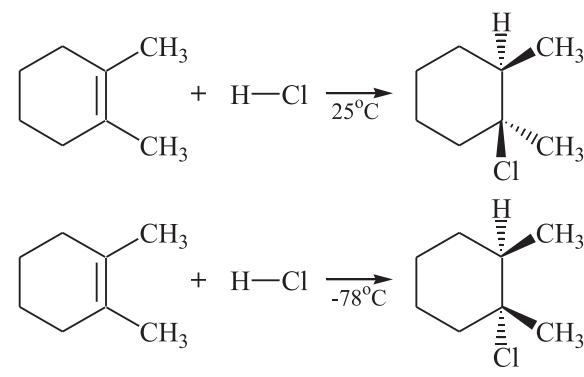

Esquema 5

Mecanismo concertado

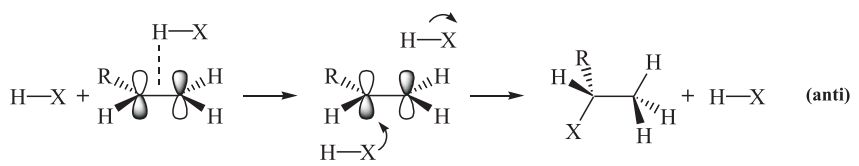

Esquema 6

Mecanismo iônico

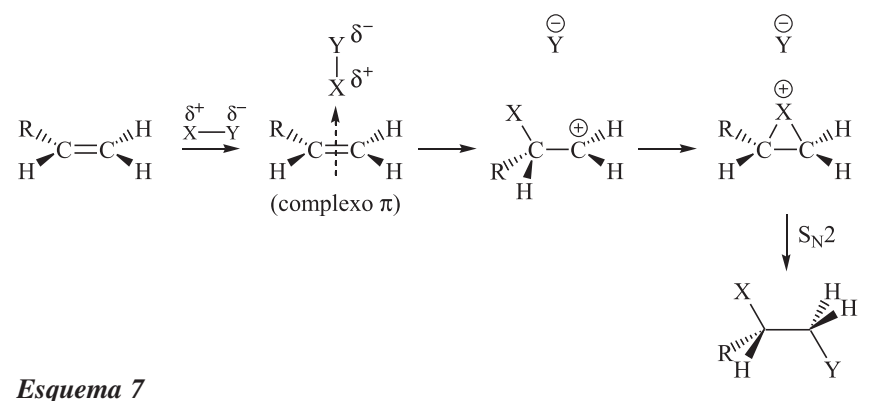

A adição de $\mathrm{HBr}$ a um alqueno assimétrico pode ser complicada devido à competição entre processos de adição iônico e radicalar. Isto ocorre se o reagente estiver contaminado com impurezas, como, por exemplo, peróxido de hidrogênio, ou se a reação for feita na presença de luz. Neste caso ocorre a formação de produto de adição anti-Markovnikov. Por exemplo (Esquema 8):

$$
\mathrm{R}-\mathrm{CH}=\mathrm{CH}_{2}+\mathrm{H}-\mathrm{Br} \underset{\text { ou luz }}{\stackrel{\mathrm{H}_{2} \mathrm{O}_{2}}{\longrightarrow}} \mathrm{R}-\mathrm{CH}_{2}-\mathrm{CH}_{2}-\mathrm{Br}
$$

\section{Esquema 8}

Adição de $\mathrm{H}-\mathrm{X}$ a alquenos assimétricos ocorre com mudança de estereoquímica do produto, quando um dos carbonos da ligação insaturada está ligado a um grupo que permite a extensão da conjugação. Neste caso, o produto da adição tem estereoquímica syn e a formação deste produto é explicada por um mecanismo que envolve a formação de um par iônico, compatível com um processo bimolecular que segue uma lei de velocidade de segunda ordem (Esquema 9).

$\mathrm{A}$ adição de $\mathrm{X}-\mathrm{Y}$ a dienos isolados e acumulados pode ocorrer com a adição de um ou dois mols do reagente, de acordo com a regra de Markovnikov (Esquema 10).

\section{Adição a dienos conjugados}

A adição de reagente do tipo $\mathrm{X}-\mathrm{Y}$ a dienos conjugados pode

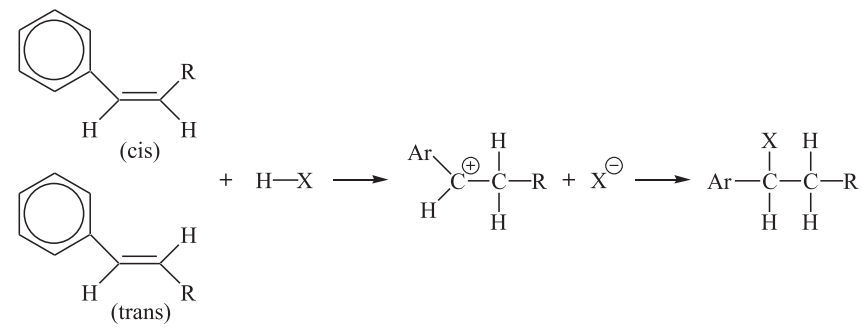

Esquema 9

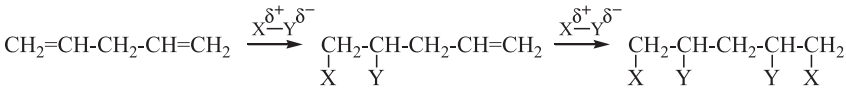

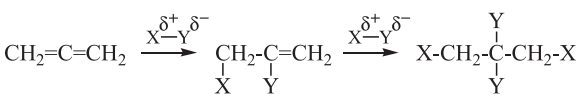

Esquema 10

ocorrer levando à formação de produtos de adição 1,2 ou 1,4 (Esquema 11). O tipo de produto obtido depende da temperatura e do solvente em que a reação é realizada. Temperatura alta favorece a formação do produto de adição 1,4 (nestas condições, diz-se que a reação ocorre com controle termodinâmico); temperatura baixa favorece o produto de adição 1,2 (e a reação é dita ocorrer com controle cinético).

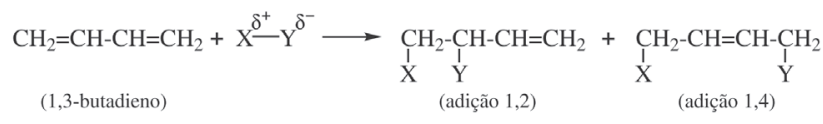

\section{Esquema 11}

Existem evidências de que a reação ocorre através de um mecanismo iônico, envolvendo a formação de um cátion alílico intermediário (Esquema 12).

\section{Mecanismo iônico}

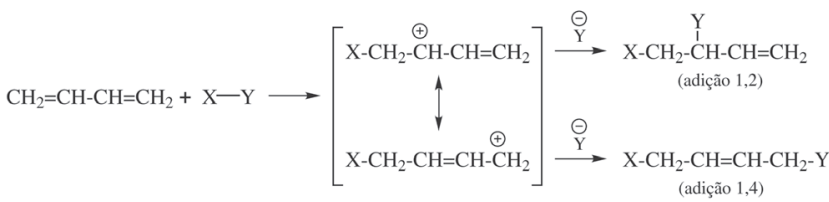

Esquema 12

Um exemplo de reação de adição a dienos conjugados é a adição de cloreto de deutério ao trans-1,3-pentadieno. ${ }^{13}$ Esta reação pode ser feita na ausência de solvente ou na presença de pentano, ácido acético deuterado ou nitrometano, produzindo uma mistura de trans-4-cloro-2-penten-5-d e trans-4-cloro-2-penten-1-d. Experimentalmente, constata-se que o produto de adição 1,2 predomina e que sua formação segue a regra de Markovnikov. Além disso, existem evidências experimentais de que esta reação ocorre segundo um mecanismo que envolve a formação de um par iônico, o que é compatível com um processo bimolecular (Esquema 13).

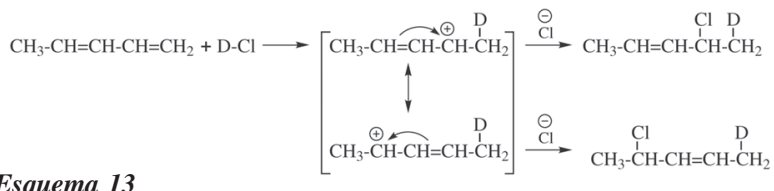




\section{RESULTADOS E DISCUSSÃO}

\section{Adição a alquenos não-conjugados}

A reação de adição de ácido clorídrico a propeno $(\mathrm{HCl}$ $+\mathrm{CH}_{2}=\mathrm{CHCH}_{3}$ ) foi escolhida como reação-modelo para o estudo computacional do mecanismo.

As entradas para os cálculos foram preparadas durante a aula expositiva e logo após a explicação sobre os fundamentos da regra de Markovnikov e sua aplicação para a reação em questão. Para a obtenção da energia do reagente foi realizado um cálculo de otimização da geometria dos fragmentos $\mathrm{CH}_{2}=\mathrm{CHCH}_{3}$ e $\mathrm{HCl}$, considerando a aproximação da supermolécula (fragmentos separados por uma distância muito grande, cerca de $50 \AA$ ). Foram mostrados alguns recursos importantes para a manipulação individual dos fragmentos, tais como as teclas "ALT+CTRL" e "ALT+SHIFT", para rotacionar e translacionar um fragmento mantendo o outro fixo, respectivamente. A entrada para a otimização do produto foi construída a partir da geometria do reagente, para garantir a mesma numeração e conectividade entre os átomos. A energia do produto foi obtida para a geometria otimizada.

Após a obtenção das energias e estruturas dos reagentes $\left(\mathrm{CH}_{2}=\mathrm{CHCH}_{3}\right.$ e $\left.\mathrm{HCl}\right)$ e do produto, foram mostrados detalhes da funcionalidade dos procedimentos de busca pela estrutura do estado de transição, implementados no programa Gaussian (QST2 e QST3). Para o caso do mecanismo via Markovnikov, o cálculo a partir das estruturas dos reagentes e do produto, com a opção QST2, foi suficiente para a obtenção do estado de transição. No entanto, para o mecanismo anti-Markovnikov foi preciso utilizar uma estrutura inicial como "chute" para o estado de transição, realizando-se um cálculo QST3. Depois da finalização desta etapa, o resultado obtido para a estrutura do estado de transição foi confirmado mediante a análise dos modos normais de vibração. Um único modo normal de frequiência imaginária, correspondendo à quebra da ligação $\mathrm{H}-\mathrm{Cl}$ e formação das ligações $\mathrm{C}-\mathrm{Cl}$ e $\mathrm{C}-\mathrm{H}$, confirmou que a coordenada obtida era a desejada. O caminho de reação, a partir do estado de transição, foi também confirmado através do cálculo IRC. Os resultados para as energias relativas são mostrados na Figura 1.

Como pode ser observado na Figura 1, o mecanismo considerando a regra de Markovnikov é favorecido cinética (menor ener-

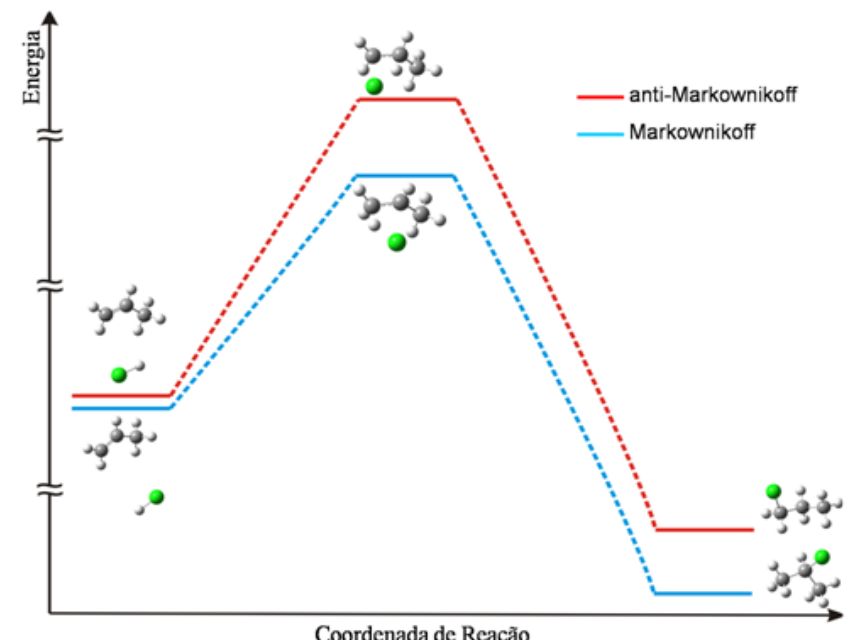

Figura 1. Diagrama qualitativo mostrando a coordenada da reação $\mathrm{CH}_{2}=\mathrm{CHCH}_{3}+\mathrm{HCl}$ via Markovnikov e anti-Markovnikov. Note que são usadas diferentes escalas para as energias relativas dos reagentes, produtos e estados de transição gia de ativação) e termodinamicamente (menor energia do produto). Dessa forma, espera-se que o estudante agora seja capaz de discutir esta regra fundamental da química orgânica baseando-se nos aspectos energéticos das espécies envolvidas.

\section{Adição a dienos conjugados}

Conforme discutido anteriormente, o produto da adição a dienos conjugados pode ser do tipo 1,2 ou 1,4, dependendo das condições em que a reação se processa. Para entender este mecanismo, estudou-se a reação de adição do ácido clorídrico a 1,3-butadieno $\left(\mathrm{HCl}+\mathrm{CH}_{2}=\mathrm{CHCH}=\mathrm{CH}_{2}\right)$. $\mathrm{O}$ objetivo foi investigar a estabilidade relativa dos adutos 1,2 e 1,4 .

Um aspecto importante desta reação é a conformação do 1,3butadieno. Sabe-se que esta molécula pode apresentar as conformações $s$-cis e $s$-trans, sendo a última ligeiramente mais estável que a primeira (cerca de $3 \mathrm{kcal} / \mathrm{mol}$ ). ${ }^{6}$ Estas duas possibilidades conformacionais para o reagente foram consideradas nos cálculos. Dessa forma, as seguintes reações foram estudadas:

$i$ trans-1,3-butadieno $+\mathrm{HCl} \rightarrow$ 3-cloro-but-1-eno [adição 1,2];

ii trans-1,3-butadieno $+\mathrm{HCl} \rightarrow$ 1-cloro-but-2-eno [adição 1,4];

iii $\quad$ cis-1,3-butadieno $+\mathrm{HCl} \rightarrow$ 3-cloro-but-1-eno [adição 1,2];

iv $\quad$ cis-1,3-butadieno $+\mathrm{HCl} \rightarrow$ 1-cloro-but-2-eno [adição 1,4 ].

Todas as etapas discutidas anteriormente foram utilizadas para as quatro reações. Para as adições 1,2 a busca pelo estado de transição foi realizada utilizando-se o método QST2, enquanto que a adição 1,4 exigiu um cálculo QST3. Os resultados são mostrados na Figura 2.

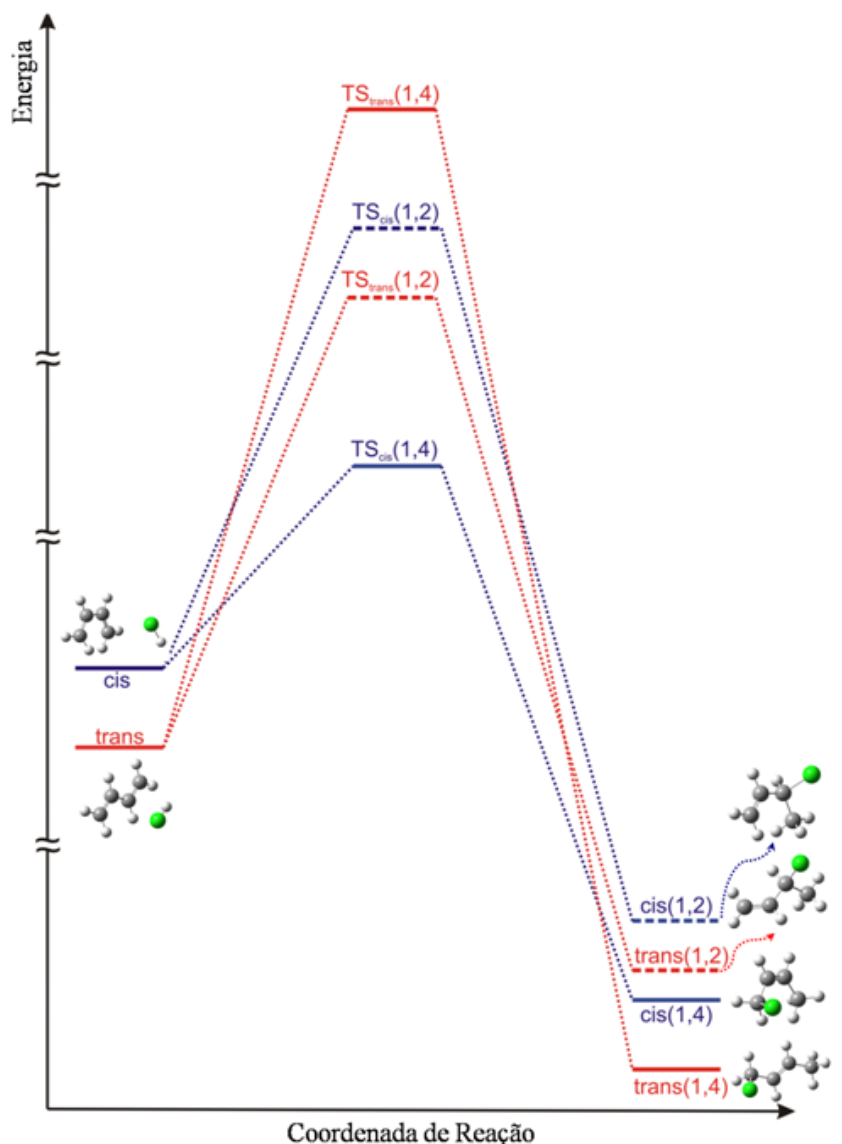

Figura 2. Diagrama qualitativo mostrando a coordenada da reação de adição $\mathrm{CH}_{2}=\mathrm{CHCH}=\mathrm{CH}_{2}+\mathrm{HCl}$. Note que são usadas diferentes escalas para as energias relativas dos reagentes, produtos e estados de transição 
Considerando que o presente trabalho parte do princípio de que a reação estudada está em fase gasosa, espera-se que o mecanismo envolvido seja concertado, compatível com o processo bimolecular. Dessa forma, o estado de transição é possivelmente uma estrutura cíclica e a adição é do tipo syn. Analisando-se os resultados mostrados na Figura 2, pode-se constatar que a adição 1,4 é favorecida termodinamicamente, sendo o produto trans-1-cloro-but-2-eno mais estável que o seu isômero cis. Entretanto, observa-se que a barreira de ativação para a adição 1,4 do $\mathrm{HCl}$ ao 1,3-butadieno quando na conformação $s$-cis é consideravelmente menor que a da correspondente reação na conformação s-trans. Esta diferença de energia entre os estados de transição pode ser explicada considerando-se que, na primeira reação, o estado de transição se assemelha a um anel de 6 membros na conformação de barco, semelhante ao estado de transição de uma ciclo-adição do tipo Diels-Alder, ${ }^{14}$ conforme Esquema 14. Neste caso, a estrutura do estado de transição é simétrica e o mecanismo é controlado pela regra de WoodwardHoffman, que é fundamentada na conservação da simetria dos orbitais. No entanto, para a segunda reação, o estado de transição não é simétrico e, portanto, não é correto falar de "simetria dos orbitais".

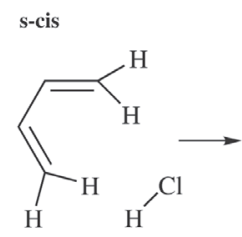<smiles>ClCCCCCCCl</smiles><smiles>C/C=C\CCl</smiles><smiles>C=CCl</smiles><smiles>CC=CC=CC</smiles><smiles>C/C=C/[CH]Cl</smiles>

Esquema 14

Nas Figuras 3a e 3b são mostrados os orbitais de ligação, relativos ao estado de transição, para as duas reações de adição 1,4 do $\mathrm{HCl}$ ao 1,3-butadieno envolvendo as conformações cis e trans. É possível observar que o conjunto de orbitais na conformação $s$-cis é favorecida pela "distribuição espacial" dos orbitais, o que não acontece com a reação envolvendo o 1,3-butadieno na conformação $s$-trans. Esta distribuição espacial, que no caso da reação de Diels-Alder é controlada por simetria, diz respeito aos seguintes aspectos: os orbitais de ligação $\pi$ do butadieno foram deslocados das posições 1,3 para a posição 2 , no caso da conformação cis, o que não acontece para a conformação trans; os orbitais $\sigma$ e os correspondentes anti-ligantes referentes as ligações $\mathrm{Cl}-\mathrm{C}$ e $\mathrm{H}-\mathrm{C}$ foram parcialmente formados para o caso cis, o que também não ocorre para a conformação trans. Nas Figuras 3a e 3b a notação adotada é a seguinte: LUBO diz respeito aos orbitais de ligação virtuais de mais baixa energia, à semelhança dos orbitais LUMO, e HOBO diz respeito aos orbitais de ligação ocupados de mais alta energia, à semelhança dos orbitais HOMO.

\section{CONCLUSÕES}

No presente trabalho é descrita uma experiência pedagógica envolvendo estudantes de pós-graduação e graduação em química, na qual foram escolhidas reações clássicas de química orgânica

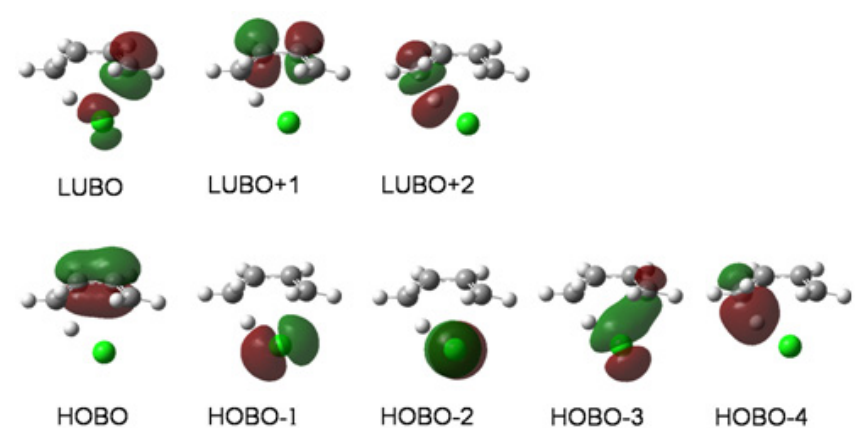

Figura 3a. Orbitais de ligação (NBO) calculados no estado de transição da reação de adição do $\mathrm{HCl}$ ao 1,3-butadieno na conformação s-cis
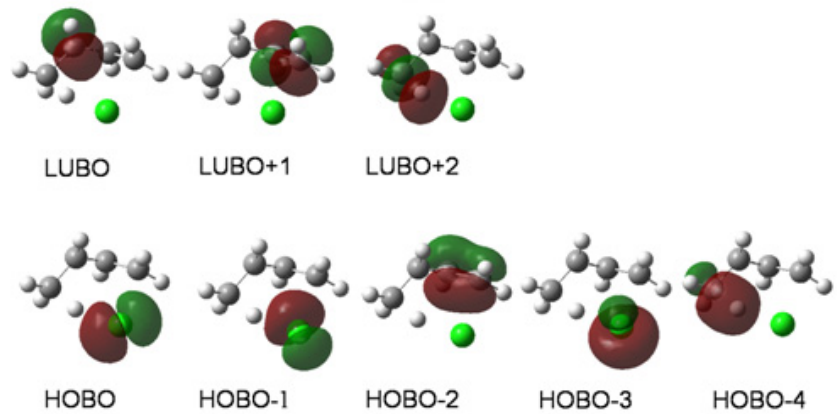

Figura 3b. Orbitais de ligação (NBO) calculados no estado de transição da reação de adição do $\mathrm{HCl}$ ao 1,3-butadieno na conformação s-trans

para serem estudadas empregando química quântica computacional. A clássica regra de Markovnikov foi o primeiro exemplo estudado. O nível do cálculo de química quântica empregado foi o mais simples possível (Hartree-Fock com a base mínima STO-3G), de modo a minimizar a demanda computacional e, dessa forma, possibilitar que todas as etapas do estudo fossem realizadas em uma única aula com duração de $2 \mathrm{~h}$. A proposta era revisitar qualitativamente conceitos fundamentais que controlam uma reação química, tendo como ferramentas um computador, algumas implementações recentes em química quântica e uma interface gráfica.

O principal desafio do trabalho foi sistematizar um procedimento que auxiliasse o professor no ensino dos mecanismos de reação. Esse procedimento, embora simplificado, ao considerar que a reação ocorre em fase gasosa e via um mecanismo bimolecular (concertado), foi suficiente para obter algumas informações importantes descritas nos livros-texto de química orgânica e que algumas vezes são bastante abstratas para o estudante de química. Por exemplo, considerando a reação de adição a alquenos não-conjugados foi possível verificar a observância à regra de Markovnikov e fundamentá-la em termos do controle reacional e aspectos regiosseletivos.

\section{AGRADECIMENTOS}

Às agências de fomento CNPq, CAPES e FAPESQ/PB pelo auxílio financeiro.

\section{REFERÊNCIAS}

1. Turro, N. J.; J. Chem. Educ. 2005, 82, 1292.

2. March, J.; Advanced Organic Chemistry - Reactions, Mechanisms and Structure $3^{\text {rd }}$ ed, Wiley Interscience, ??, 1985; Gibson, G. W.; Mastering Organic Chemistry - A Problem, Solving Approach, S.G.Sunbursch: New York, 1972.

3. Lacerda Júnior, V.; Oliveira, K. T.; Costa e Silva, R.; Constantino, M. G.; 
Silva, G. V. J.; Quim. Nova 2007, 30, 727

4. Ventura, E.; Dallos, M.; Lischka, H.; J. Chem. Phys. 2003, 118, 1702.

5. Ventura, E.; Monte, S. A.; Dallos, M.; Lischka, H.; J. Phys. Chem. A 2003, 107,1175

6. Ventura, E.; Dallos, M.; Lischka, H.; J. Chem. Phys. 2003, 118, 10963.

7. Lischka, H.; Ventura, E.; Dallos, M.; Chem. Phys. Chem. 2004, 5, 1365.

8. Frisch, M. J.; Trucks, G. W.; Schlegel, H. B.; Scuseria, G. E.; Robb, M. A.; Cheeseman, J. R.; Zakrzewski, V. G.; Montgomery, J. A.; Stratmann Jr., R. E.; Burant, J. C.; Dapprich, S.; Millam, J. M.; Daniels, A. D.; Kudin, K. N.; Strain, M. C.; Farkas, O.; Tomasi, J.; Barone, V.; Cossi, M.; Cammi, R.; Mennucci, B.; Pomelli, C.; Adamo, C.; Clifford, S.; Ochterski, J. Petersson, G. A.; Ayala, P. Y.; Cui, Q.; Morokuma, K.; Salvador, P.; Dannenberg, J. J.; Malick, D. K.; Rabuck, A. D.; Raghavachari, K.; Foresman, J. B.; Cioslowski, J.; Ortiz, J. V.; Baboul, A. G.; Stefanov, B. B.; Liu, G.; Liashenko, A.; Piskorz, P.; Komaromi, I.; Gomperts, R.; Martin, R. L.; Fox, D. J.; Keith, T.; Al-Laham, M. A.; Peng, C. Y.; Nanayakkara,
A.; Challacombe, M.; Gill, P. M. W.; Johnson, B.; Chen, W.; Wong, M. W.; Andres, J. L.; Gonzalez, C.; Head-Gordon, M.; Replogle, E. S.; Pople, J. A.; Gaussian 98W; Revision A.1; Gaussian: Pittsburgh, PA, 2001.

9. Foresman, J. B.; Frisch, A.; Exploring Chemistry with Electronic Structure Methods: A Guide to Using Gaussian, Gaussian Inc.: Pittsburgh, 1993.

10. Gonzalez, C.; Schlegel, H. B.; J. Chem. Phys. 1989, 90, 2154; Gonzalez, C.; Schlegel, H. B.; J. Phys. Chem. 1990, 94, 5523.

11. Roothan, C. C. J.; Rev. Mod. Phys. 1951, 23, 69; Szabo, A.; Ostlund, N. S.; Mod. Quantum Chem. 1982, McGraw-Hill: New York, 1982.

12. Hehre, W. J.; Stewart, R. F.; Pople, J. A.; J. Chem. Phys. 1969, 2657.

13. Nordland, J. E.; Owuor, P. O.; Haky, J. E.; J. Am. Chem. Soc. 1979, 101, 1288 .

14. Tardy, D. C.; Ireton, R.; Gordon, A. S.; J. Am. Chem. Soc. 1979, 101, 1508; Uchiyama, M.; Tomioka, T.; Amano, A.; J. Phys. Chem. 1964, 68, 1878; Diau, E. W.-G.; De Feyter, S.; Zewail, A. H.; Chem. Phys. Lett. 1999, 304 , 134. 